\title{
Evaluation of Antenna Placement in Urban-Road Scenarios on Beam Alignment of 5G Millimeter-Wave Small Cells
}

\author{
Indar Surahmat ${ }^{1, *}$
}

\author{
${ }^{1}$ Department of Electrical Engineering, Universitas Muhammadiyah Yogyakarta \\ ${ }^{*}$ Corresponding author. Email: indar.surahmat@umy.ac.id
}

\begin{abstract}
The use of millimeter-wave for $5 \mathrm{G}$ connections seems ready to be implemented. The high-path loss problem as a consequence is solved by the use of antenna arrays and the reduction of cell coverage with a small cell concept. Aligning beams to the best directions is then a selected concept of antenna arrays implementation. Numerous research has addressed the concept to obtain optimal beam alignments. In addition to those, this paper investigates the impacts of antenna positions to the beam alignment in urban road scenarios. Optimizing Line of Sight (LOS) connections and investigating Nonline of Sight (NLOS) are two concerns in the scenarios. A straight road is chosen to be an example of small cell implementation in an urban environment. Furthermore, the main goal of this paper is to characterize LOS and NLOS connection for proposed antenna positions i.e. wall-mounted and lamppost scenarios. These scenarios are then carefully evaluated. The results show that the installation of the antenna on the lamppost gains more LOS connections compared to that in the wall-mounted scenario. Its installation improves EM field strength by around $5 \mathrm{~dB}$. In addition, the basic concept of LOS and NLOS in terms of connection due to the use of antenna arrays should be modified. A user in the LOS region is potentially served by an NLOS connection.
\end{abstract}

Keywords-5G, millimeter wave, beam alignment, urban, small-cell

\section{INTRODUCTION}

The use of millimeter-wave for enhancing capacities of $5 \mathrm{G}$ connections is thoroughly studied. It is related to the availability of wide bandwidth which is not used by other purposes, unlike that in frequencies lower than $6 \mathrm{GHz}$ [1]-[7]. Unfortunately, a higher path loss is a drawback that must be considered. Growing research regarding this issue proposes some solutions, two of them are discussed in this paper. First, the use of antenna arrays with beamforming can enhance antenna gain to compensate for the loss. Second, a small-cell will serve a smaller [8] and extended coverage [9].

Applying beamforming with antenna arrays especially large- or massive-arrays results a coverage of a single beam produced by arrays have a narrower beamwidth as well as less in covered areas. Unlike conventional antennas that cover wide areas with beamwidth from $45^{\circ}$ to $120^{\circ}$, largearrays have much smaller beamwidth less than $10^{\circ}$ that depend on the size of arrays. The greater number of arrays produces the narrower beamwidth.

Some papers have described about beam alignments. The important points from those are maximizing LOS connections and reducing time used for searching optimal beams. For instance, an optimum beam is gained from a measurement of a reference signal in a downlink channel that reported back to the transmitter [10], by employing a specific algorithm to ensure less in beam searching complexity [11].
Considering small-cells implementation, antennas in millimeter wave will be installed denser and closer to the users. The idea explored in this study is started from the potential of antenna-installation positions proposed in [4] which then discussed later in this paper. To show the method that proposed in the study, this paper presents a single case of small-cells in the urban environment.

A straight road surrounded by buildings is modeled for the evaluation purpose. On the discussion, this study rather focuses on characterizing the pattern of LOS and NLOS than discusses the accuracy of simulation results.

The rest of this paper is described as follows. In part II, step by step of the evaluation methods are explained. Part III provides the results of the methods specified and discusses the findings. Summaries and potential future works are described in the last part.

\section{METHODS}

The study establishes the methods including some aspects that described as follows.

\subsection{Antenna Arrays}

The use of antenna arrays in the $5 \mathrm{G}$ millimeter wavestill becomes one of the important topics addressed by some researcher. One of the topics that has relation with this study is the maximum scanning angle that when the scanning angle 
larger than $40^{\circ}$, the gain performance degrades as mentioned in [3].

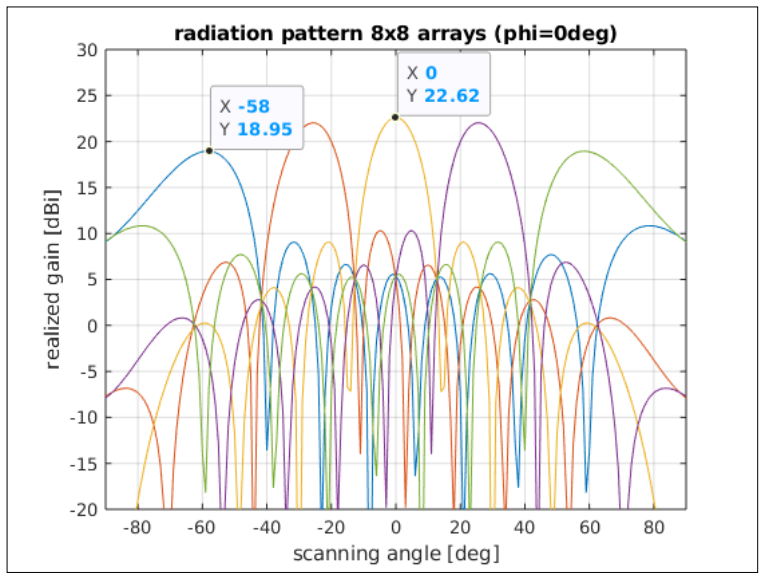

Figure 1 Overview gain performance of URA antennas horizontal scanning

In the evaluation, an $8 \times 8$ uniform rectangular arrays is used with patch antenna as a single element. Figure 1 shows antenna arrays radiation pattern in a variety of scanning angles. In the simulation, the patterns are simply obtained by adjusting phase shift $\delta$ that follows Equation (1) where $\psi$ is beam edge angle, $\mathrm{k}$ is wave number, $\mathrm{d}$ is spacing between elements and $\theta$ is radiation angle measured from z-axis. While in uniform rectangular arrays, two-phase shifts in the direction of $\theta$ and $\varphi$, where $\varphi$ is the radiation angle measured from y-axis, form 3D scanning angles.

$\delta=\psi-k d \cos (\theta)$

In the interval of $30^{\circ}$ figured in Figure 1, the gain performance degrades about $4 \mathrm{~dB}$ at scanning angle $60^{\circ}$. Therefore, this study limits $60^{\circ}$ for the maximum scanning angle as well as the maximum Angle of Departure (AoD).

\subsection{Evaluation Scenarios}

In urban environment, a typical road corridor is chosen. As previously mentioned, the study adopts a proposal of antenna installation for small cells scenarios as in [4]. Figure 2 provides illustration of proposed antenna implementation in the small cell especially to cover a street corridor in urban roads.

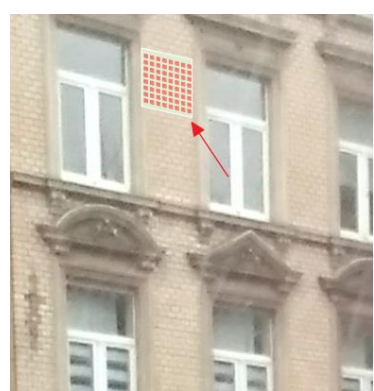

(a) Wall mounted

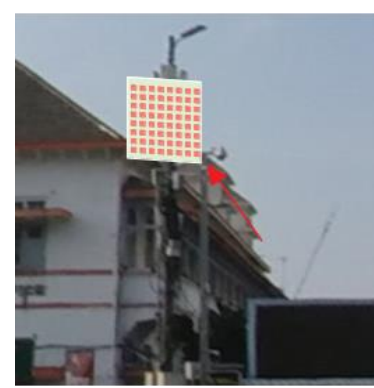

(b) Lamppost
Figure 2 Illustration of proposed antenna installation

\subsubsection{Wall mounted installation}

In a wall-mounted installation, the antenna is located on the wall side. It is installed on a selected building that potentially covers the whole street corridor as seen in Figure 2a. This is the basic consideration in coverage planning. The study places the antenna in the middle building along the street corridor.

\subsubsection{Lamppost installation}

Another growing concern of the antenna placement in the field deployment is by installing the antenna on a lamppost or other street properties [12]. For the second evaluation, the study uses a lamppost located on the street corner as shown in the Figure $2 b$.

\subsection{Ray Tracing Simulation}

Ray tracing simulation for the scenarios is then used. The research applies antenna arrays in the simulation, examines all possible scanning angles to get overall views of the best reception on a user's side. The scanning angle interval used in the simulation is $5 \mathrm{o}$. The evaluation, further, focuses on horizontal scanning angles instead of vertical scanning angles since the height of the antenna is close to the ground. The parameters used in the simulation are summarized in Table 1.

Table 1. Simulation parameters of the scenarios

\begin{tabular}{|l|l|}
\hline \multicolumn{1}{|c|}{ Parameter } & \multicolumn{1}{c|}{ Value } \\
\hline Frequency $(\mathrm{GHz})$ & 28 \\
\hline Transmitter & \\
\hline Antenna Type & $8 \times 8 \mathrm{URA}$ \\
\hline Transmit Power $(\mathrm{dBm})$ & 50 \\
\hline Antenna Height $(\mathrm{m})$ & 7 \\
\hline Receiver & \\
\hline Antenna Type & Omnidirectional \\
\hline Antenna Height $(\mathrm{m})$ & 15 \\
\hline
\end{tabular}

The overall design of the scenarios simulated in the ray tracing is illustrated in Figure 3. A receiver moves from the starting point along the street corridor. The selected points have interval $1 \mathrm{~m}$, while the resolution is $0.5 \mathrm{~m}$. 


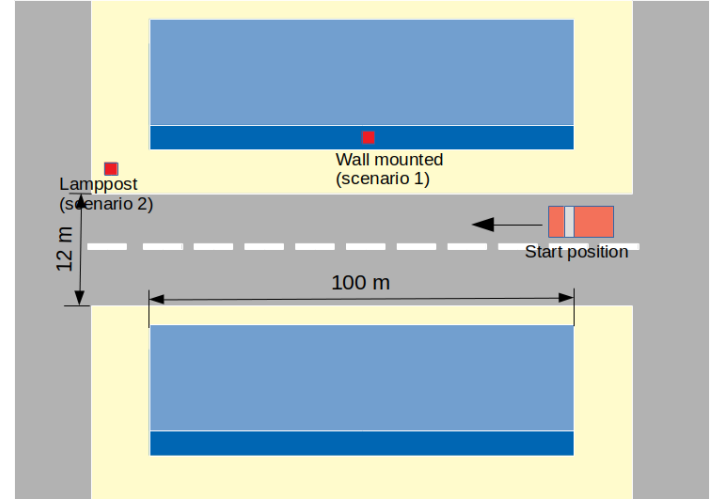

Figure 3 Overall evaluation scenario

\section{RESULT AND DISCUSSION}

\subsection{Wall Mounted Antenna Scenario}

Figure 4 shows simulation result of the wall-mounted installation scenario. From the separation of LOS components, Figure 4b, it can be seen that a user that moves along the street mainly will be served by NLOS connection. The intensity of the received NLOS signal will depend on the reflection parameter of the wall surrounding the road. Figure $4 \mathrm{~b}$ also shows the potential points where a mobile user will be served by the LOS connection. This explains that the implementation of antenna arrays have a consequence in limiting LOS connection since the antenna beam has a much narrower range.

Beam alignment process evaluates the best AoD based on the best EM field at the receiver's side. Then, receivers usually send feedback reports on their measurements. If the best value of the EM field is chosen, information on the scanning angle can be extracted as seen in Figure 5. Since the antenna position is located in the middle of the street corridor, the LOS connection can cover up to $10 \mathrm{~m}$ range from the wall-mounted antenna, while other positions are served by NLOS connection. Applying antenna with wider scanning range which is also the hot topic in antenna-arrays design possibly improves the limitation of the LOS connection region in this scenario.

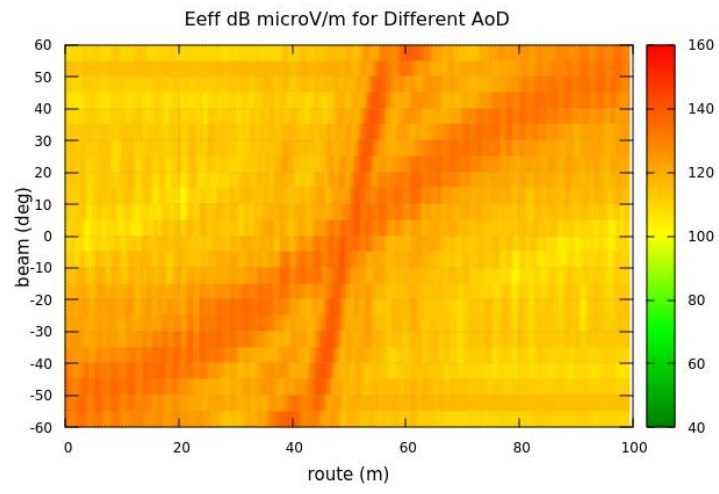

(a) LOS-NLOS

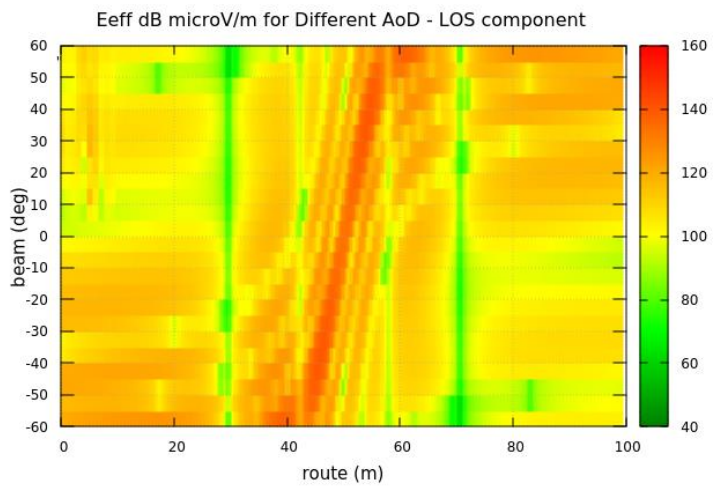

(b) LOS Component

Figure 4 LOS and NLOS pattern for all scanning angles in wall mounted scenario

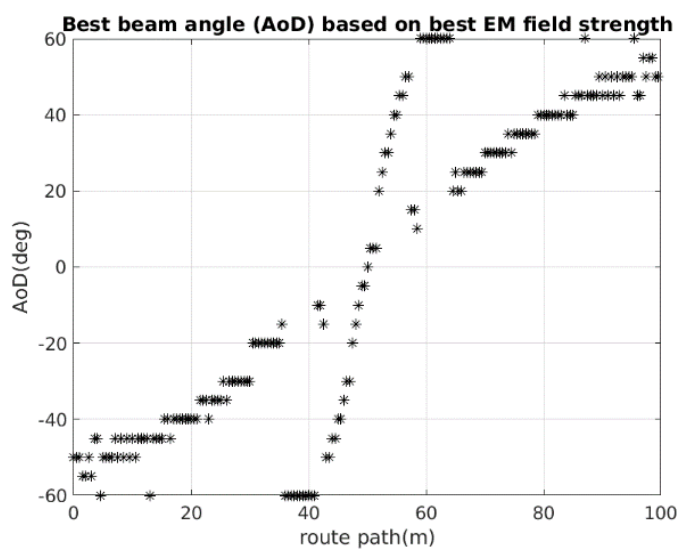

Figure 5 Optimal scanning angles based on the best EM field strength in wall mounted scenario

From the NLOS profile, the study draws attention to the building opposite of the selected building for antenna installation. By comparing between Figure 4a and Figure 4b, subtracting the LOS component, the NLOS connection particularly is supported by the reflection signals from the building in front of the antenna. The exploration of building materials in connection with reflections or scatterings is required to estimate more precise coverage prediction.

\subsection{Lamppost Scenario}

In the lamppost scenario, the simulation exhibits different beam patterns. Since the installed antennas face the corridor, there is no significant change of the scanning angle along the corridor, almost $80 \%$ of the scanning angle located in the range $\pm 10^{\circ}$. Figure 6 illustrates the condition. In other words, with this scenario, the scanning angle can be kept constantly along the corridor and beam alignment process should be much less complicated.

The drawback of this scenario is when LOS connection is not present, subtracting the LOS component in Figure $6 \mathrm{~b}$ from Figure $6 \mathrm{a}$, there are some chances of NLOS connection. Figure 6a shows three alternatives of NLOS connection instead of the LOS one. Those conditions mean the process of beam searching will take a longer time. Prioritizing the 
beam scanning angles could be a solution. The system may set those alternatives into a sequential rank.

When the optimal EM field strength is taken, Figure 7 exhibits the best horizontal scanning angles. It emphasizes previous mentioned evidences that mainly connection is LOS.

\subsection{Comparison}

It is interesting then by the use of cumulative distribution function (CDF) of EM field strength, the two scenarios are compared. The comparison uses an assumption that both scenarios correctly apply the best AoD along the street corridor. Fig. 8a shows the EM field strength along the route, while fig. $8 \mathrm{~b}$ presents cumulative distribution of the EM field strength. This result shows that in a straight road corridor, installing the antenna arrays in a lamppost provides users a higher opportunity to obtain a better signal strength by approximately $5 \mathrm{~dB}$.

\section{CONCLUSION AND FUTURE WORK}

Investigation of antenna position in urban road scenarios has been presented. Both proposed antenna positions show different characteristics. The installation on the building wall has limitations on LOS connection and the NLOS alternative depends on the buildings in front of the antenna. While in the lamppost scenario, it provides a better LOS connection. When the LOS connection is disappeared, this scenario increases the beam searching complexity. The EM field strength of the lamppost scenario is superior to the wallmounted scenario about $5 \mathrm{~dB}$.

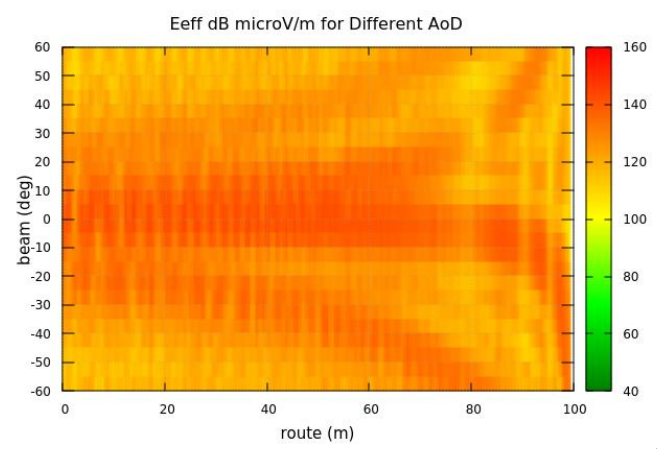

(a)

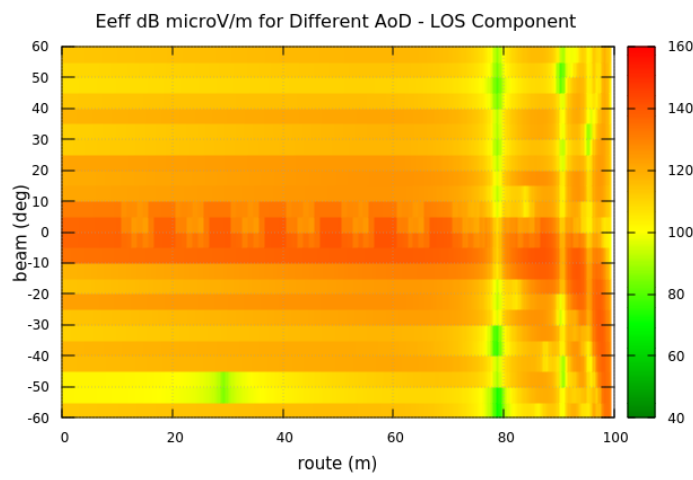

(b) LOS Component

Figure 6 LOS and NLOS pattern for all scanning angles in lamppost scenario

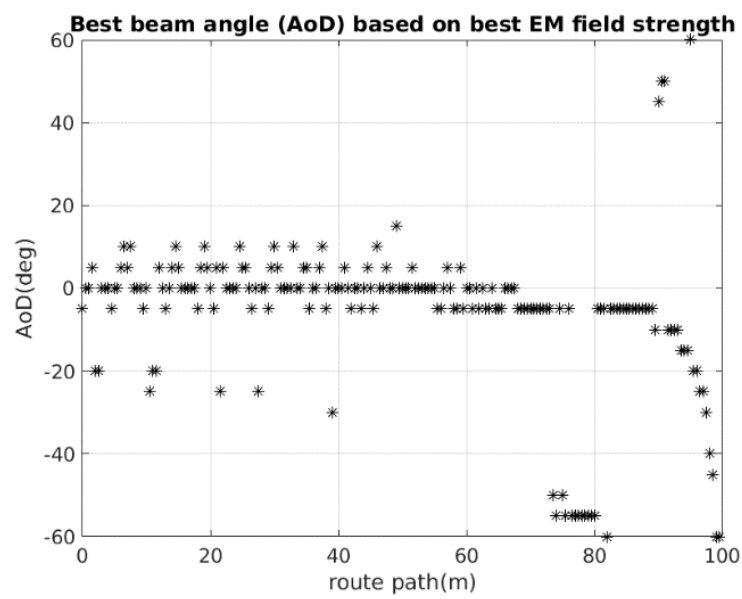

Figure 7 LOS and NLOS pattern for all scanning angles in lamppost scenario

The aforementioned evaluation promotes the shift of the LOS and NLOS concept. It should be noted that although there is no obstacle between the transmitter and the receiver based on their position, the LOS in terms of position, the connection between them is not always LOS. From the evaluation, future works may explore the impact of scatterers close to a user. This proposed method provide a clear view of the impact. By adding random scatterers at some objects may also improve the limitation on reflection analyzed in this study. 


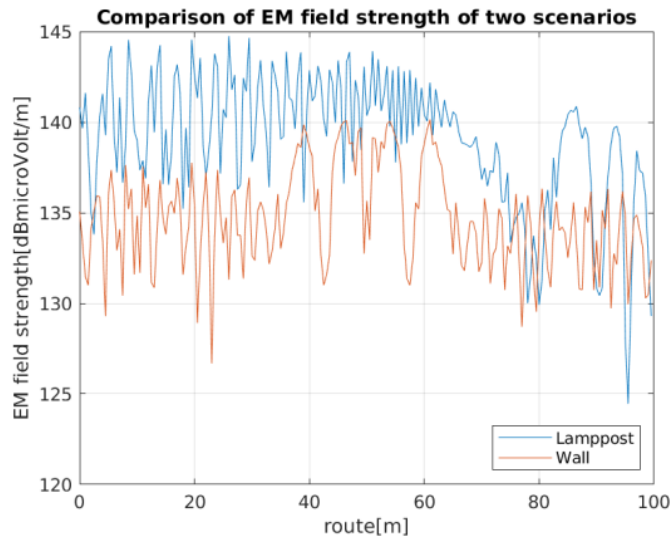

(a) EM field strength

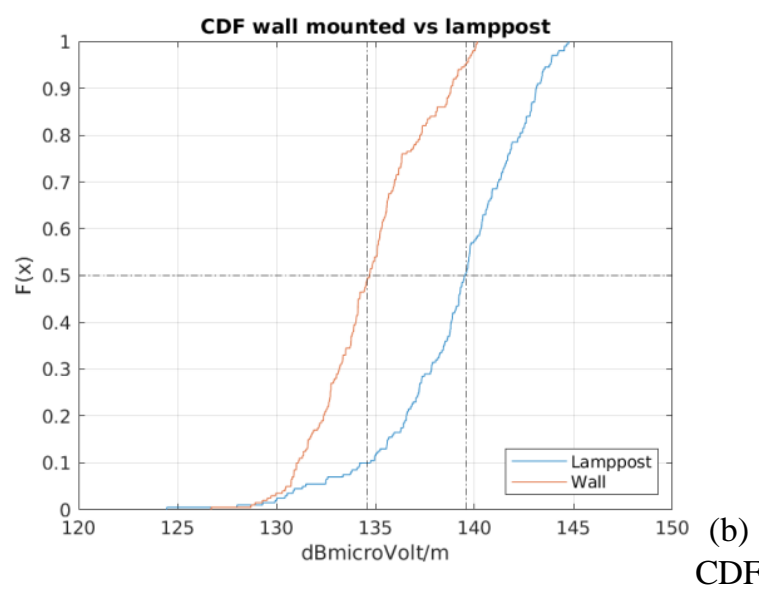

EM field strength

Figure 8 Comparison EM field strength of optimum horizontal scanning angle

\section{AUTHORS' CONTRIBUTIONS}

Strictly speaking, what our focus in here is to analyse some potential of antenna installations in the mmwave $5 \mathrm{G}$ recommended by interested stake holders in this field. We propose an approach on how to develop the purpose mainly by two steps. First, we set up an environment and run a ray tracing simulation with a concept which has been details explained in the method section. Second, we build investigation based on the output of the simulation.

\section{ACKNOWLEDGMENTS}

The author would like to thank to Universitas Muhammadiyah Yogyakarta which has supported the study.

\section{REFERENCES}

[1] S. Salous et al., "Millimeter-Wave Propagation: Characterization and modeling toward fifth-generation systems. [Wireless Corner]," in IEEE Antennas and Propagation Magazine, vol. 58, no. 6, pp. 115-127, Dec. 2016, doi: 10.1109/MAP.2016.2609815.

[2] J. Lee, J. Lee and S. Kim, "Improving the Accuracy of Millimeter-Wave Ray-Tracing Simulations by Modeling
Roadside Trees," in IEEE Antennas and Wireless Propagation Letters, vol. 18, no. 1, pp. 162-166, Jan. 2019 , doi: 10.1109/LAWP.2018.2883633.

[3] R. Mittra, "Some Challenges in Millimeter Wave Antenna Designs for 5G," 2018 International Symposium on Antennas and Propagation (ISAP), Busan, Korea (South), 2018, pp. 12 .

[4] W. Fan et al., "A Step Toward 5G in 2020: Low-cost OTA performance evaluation of massive MIMO base stations.," in IEEE Antennas and Propagation Magazine, vol. 59, no. 1, pp. 38-47, Feb. 2017, doi: 10.1109/MAP.2016.2630020.

[5] S. Hur et al., "Proposal on Millimeter-Wave Channel Modeling for 5G Cellular System," in IEEE Journal of Selected Topics in Signal Processing, vol. 10, no. 3, pp. 454469, April 2016, doi: 10.1109/JSTSP.2016.2527364.

[6] J. Lee, J. Choi and S. Kim, "Cell Coverage Analysis of 28 $\mathrm{GHz}$ Millimeter Wave in Urban Microcell Environment Using 3-D Ray Tracing," in IEEE Transactions on Antennas and Propagation, vol. 66, no. 3, pp. 1479-1487, March 2018 , doi: 10.1109/TAP.2018.2797531.

[7] W. Keusgen, R. J. Weiler, M. Peter, M. Wisotzki and B. Göktepe, "Propagation measurements and simulations for millimeter-wave mobile access in a busy urban environment," 2014 39th International Conference on Infrared, Millimeter, and Terahertz waves (IRMMW-THz), Tucson, AZ, 2014, pp. 1-3, doi: 10.1109/IRMMW-THz.2014.6955989.

[8] F. Al-Ogaili and R. M. Shubair, "Millimeter-wave mobile communications for 5G: Challenges and opportunities," 2016 IEEE International Symposium on Antennas and Propagation (APSURSI), Fajardo, 2016, pp. 1003-1004, doi: 10.1109/APS.2016.7696210.

[9] T. A. Nugraha, I. Surahmat and F. Firdaus, "Block diagonalization precoding and power allocation for clustering small-cell networks," Bulletin of Electrical Engineering and Informatics, Vol.9, No. 6, December 2020, pp. 2364-2370, doi: $10.11591 /$ eei.v9i6.2580

[10] E. Rastorgueva-Foi, M. Costa, M. Koivisto, K. Leppänen and M. Valkama, "Dynamic Beam Selection for Beam-RSRP Based Direction Finding in mmW 5G Networks," 2018 International Conference on Indoor Positioning and Indoor Navigation (IPIN), Nantes, 2018, pp. 1-6, doi: 10.1109/IPIN.2018.8533735.

[11] A. Elshafiy, K. Rose and A. Sampath, "On Optimal Beam Steering Directions in Millimeter Wave Systems," ICASSP 2019 - 2019 IEEE International Conference on Acoustics, Speech and Signal Processing (ICASSP), Brighton, United Kingdom, 2019, pp. 4709-4713, doi: 10.1109/ICASSP.2019.8683467.

[12] B. Pitakdumrongkija, M. Ariyoshi, L. Raschkowski, S. Jaeckel and L. Thiele, "Performance Evaluation of Massive MIMO with Low-Height Small-Cell Using Realistic Channel Models," 2016 IEEE 84th Vehicular Technology Conference (VTC-Fall), Montreal, QC, 2016, pp. 1-5, doi: 10.1109/VTCFall.2016.7881157.

[13] A. Milligan, Modern Antenna Design. New Jersey, IEEE Press: Wiley-Interscience, 2005. 